\title{
Advances in the prediction of long-term effectiveness of immune checkpoint blockers for non-small-cell lung cancer
}

\author{
Sara Elena Rebuzzi ${ }^{1,2}$, Alessandro Leonetti ${ }^{1}$, Marcello Tiseo ${ }^{1,3}$ \& Francesco Facchinetti ${ }^{*, 4}$ \\ ${ }^{1}$ Medical Oncology Unit, University Hospital of Parma, 43122 Parma, Italy \\ ${ }^{2}$ Medical Oncology Unit 1, Ospedale Policlinico San Martino of Genova, 16132 Genova, Italy \\ ${ }^{3}$ Department of Medicine \& Surgery, University of Parma, 43122 Parma, Italy \\ ${ }^{4}$ INSERM U981, Gustave Roussy Cancer Campus, Université Paris Saclay, 94800 Villejuif, France \\ *Author for correspondence: francescofacchinetti2@gmail.com
}

First draft submitted: 30 June 2019; Accepted for publication: 2 July 2019; Published online: 19 July 2019 Keywords: advanced non-small-cell lung cancer $\bullet$ anti-PD-1 • anti-PD-L1 • immune-checkpoint inhibitors • im-
munotherapy •long-term outcomes

Immune checkpoint blockers (ICBs), namely programmed death-1 protein (PD-1) and programmed death ligand-1 (PD-L1) inhibitors, have dramatically changed the therapeutic landscape of advanced non-small-cell lung cancer (NSCLC). The anti-PD-1 agents nivolumab and pembrolizumab, together with the anti-PD-L1 atezolizumab are standard second-line treatment options based on the improvement in overall survival (OS) compared with docetaxel in previously treated NSCLC (CheckMate 017, CheckMate 057, Keynote-010, OAK trials) [1-4]. Moving to firstline setting, pembrolizumab is the standard front-line treatment of NSCLC patients with PD-L1 expression $\geq 50 \%$ (Keynote 024) [5]. Nonetheless, combinations of anti-PD-1/PD-L1 agents with platinum-doublet chemotherapy (Keynote-189 [6] and Keynote-407 [7]) and bevacizumab (IMpower150 [8]), as well as the association of nivolumab with the anti-CTLA-4 ICB ipilimumab (CheckMate 227) [9], are the potential new options for the first-line treatment of NSCLC.

Despite the global improvement in survival outcomes, only a limited number of patients benefits from immunotherapy, experiencing durable response and long-term OS. Historically, long-term NSCLC survivors are defined as those patients who survive longer than 2 years from the date of diagnosis [10]. On the other hand, long responders are defined as having an objective complete or partial response lasting more than 6 months [11,12].

As follow-up of the first ICB trials in NSCLC patients increases, reaching up to the fifth year, more data on long-term OS are available. The Kaplan-Meier OS estimations of the pivotal ICB studies clearly reflect the trend in terms of long-term benefit of immunotherapy in NSCLC patients $[13,14]$. The tails of ICB curves reach indeed a plateau, indicating that a non-negligible quote of patients can experience an extended survival benefit, potentially turning out in cure. Moreover, recent data on NSCLC long-term survivors and responders can shed light on the identification of potential predictive biomarkers of long-term benefit from ICBs, which still represents an unmet need of ongoing clinical research.

\section{Long-term effectiveness of ICBs in NSCLC: data from clinical trials}

To date, increasing evidence on long-term survival estimations in NSCLC is available from many ICBs trials in the pretreated and in the first-line settings (Tables $1 \& 2$ ). In order to gather the most accurate long-term survival estimations, only studies whose updates have a median follow-up ( $\mathrm{mFU}$ ) of at least 2 years are included in this overview.

\section{Pretreated NSCLC patients \\ Nivolumab}

In the early CheckMate 003 trial, enrolling patients suffering from a wide range of malignancies, 129 pretreated NSCLC patients were treated [15]. In this study, nivolumab, across different doses, obtained 1-year (1-y), 2-year 


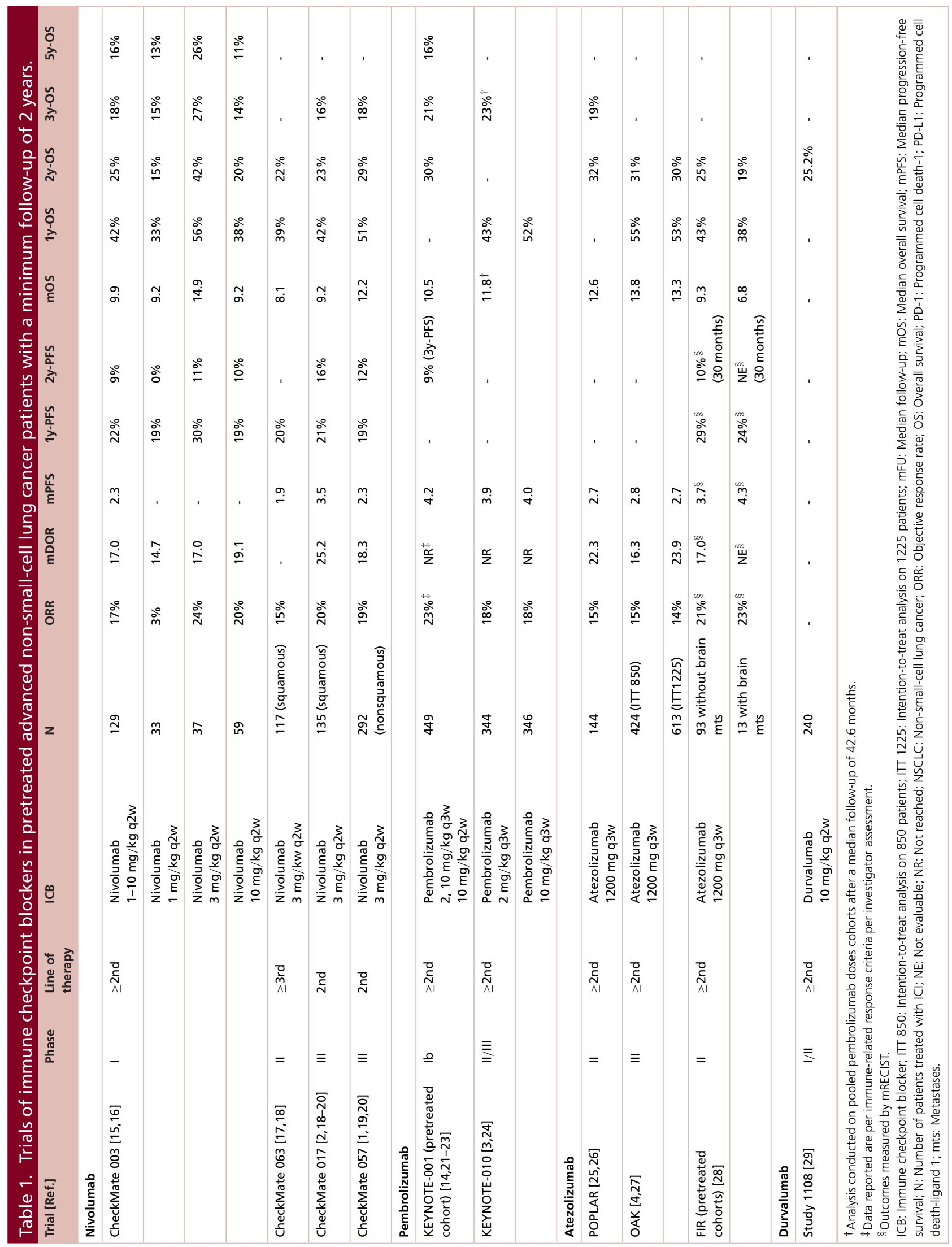




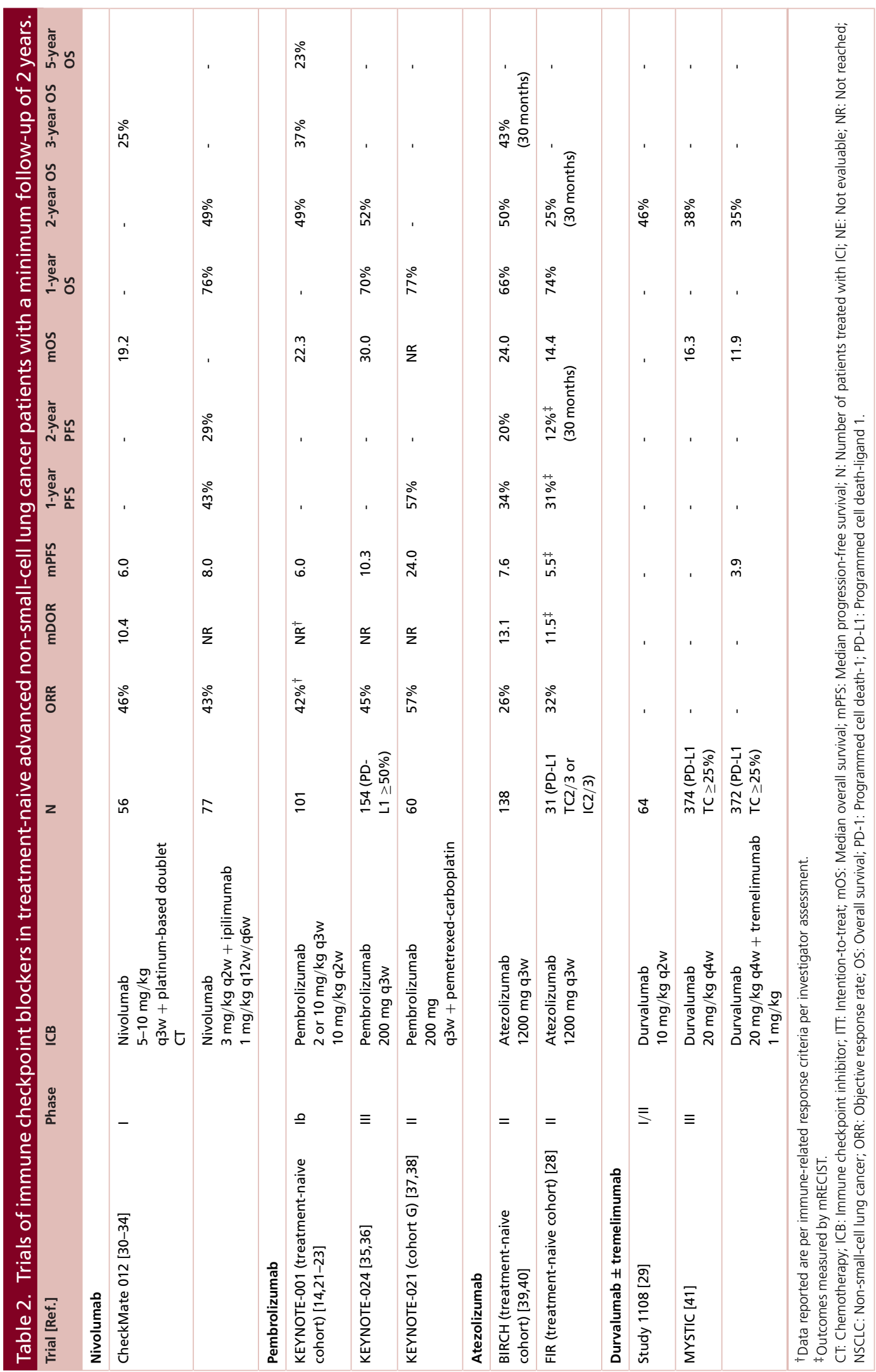


(2-y) and 3-year (3-y) OS rates of 42, 24 and 18\%, respectively [16]. Better results were obtained among the 37 patients who received nivolumab $3 \mathrm{mg} / \mathrm{kg}$, the dose chosen for further clinical development and now replaced by the flat dose of $240 \mathrm{mg}$ every 2 weeks [42]. More recently, Gettinger and colleagues published the updated results of the above-mentioned trial, based on a minimum FU of almost 5 years [13]. The median OS (mOS) was confirmed as 9.9 months, with a 5 -year $(5-y)$ OS rate of $16 \%$. Of note, a quarter of patients treated at the dose of $3 \mathrm{mg} / \mathrm{kg}$ were still alive after 5 years from nivolumab beginning [13]. Similarly, the updated CheckMate 063 Phase II trial (nivolumab in squamous NSCLC, in third or further treatment lines) reported a 1-y and 2-y OS of 39 and 22\%, respectively $[17,18]$.

In the two Phase III trials defining nivolumab as the new standard of care versus docetaxel for pretreated squamous (Checkmate 017) and nonsquamous (CheckMate 057) NSCLC patients, 1-y and 2-y OS ranged from 43 to $51 \%$ and to 23 to $29 \%$, respectively [19]. In an updated pooled analysis of the two studies, the 3-y OS was $17 \%$, confirming the survival benefit of nivolumab over docetaxel (3-y OS of 8\%) [20]. Again, when pooling the results of the four studies of nivolumab monotherapy (CheckMate 017, CheckMate 057, CheckMate 063 and CheckMate 003) 4-y OS rate was almost threefold higher when patients were treated with nivolumab compared with docetaxel (14 vs 5\%) [43].

\section{Pembrolizumab}

In the Phase I multicohort study KEYNOTE-001 pembrolizumab (2 or $10 \mathrm{mg} / \mathrm{kg}$ ) was administered to pretreated and untreated NSCLC patients with PD-L1 expressed by at least $1 \%$ of tumor cells (PD-L1 $\geq 1 \%$ ) [21]. Three updated analyses of the pretreated NSCLC cohorts were reported $[14,22,23]$. In the last one, after a mFU of 5 years, mOS was estimated at 10.5 months, with a 5 -y OS rate of $15 \%$ [14].

Besides acknowledging the superiority of pembrolizumab compared with docetaxel in NSCLC patients with PD-L1 $\geq 1 \%$ [3], the Phase II/III KEYNOTE-010 showed a 3-y OS rate for the anti-PD-1 agent of $23 \%$ (11\% for docetaxel) [24].

\section{Atezolizumab}

Among the 144 patients who received atezolizumab in the randomized Phase II POPLAR trial, 32 and 19\% were still alive at 2 and 3 years, respectively, with corresponding OS rates of 17 and 10\% in the docetaxel arm [25,26].

Similar results were observed in the long-term survival data from Phase III OAK study, with 2-y OS rates of 30 and $21 \%$ for atezolizumab and docetaxel, respectively, after a $\mathrm{mFU}$ of 28 months [27].

In addition, the FIR trial investigated atezolizumab monotherapy in chemotherapy-naive (cohort 1) and previously-treated patients (without and with brain metastases, cohort 2 and 3, respectively) who expressed PD-L1 (tumor cells $2 / 3$ [TC2/3] or immune cells $2 / 3$ [IC2/3] $\geq 5 \%$ PD-L1 staining in TC or IC) [28]. After a mFU of 33 months, the 1 -y OS and 30-month OS rate were 43 and $25 \%$ in cohort 1, 38 and $19 \%$ in cohort 2.

\section{Durvalumab}

Recently, survival updates concerning NSCLC patients included in the Phase I/II trial of durvalumab (anti-PD-L1 agent) have been published (mFU: 40 months) [29]. At 2 years since treatment beginning, 25\% of the previously treated patients were still alive.

\section{Untreated NSCLC patients Nivolumab}

The multicohort trial CheckMate 012 was the first Phase I trial designed to evaluate the potential benefit of nivolumab, given as monotherapy or in combination with other therapies, including ipilimumab and chemotherapy, for the first-line treatment of NSCLC [30,31]. The 3-y update of the three cohorts combining nivolumab with three platinum-based doublets reported an mOS of 19.2 months, with a quarter of patients still alive after 3 years [32]. The data concerning the nivolumab + ipilimumab cohort (77 patients) have also been recently updated. After 2 years, $49 \%$ of patients were alive and $29 \%$ of patients remained progression-free, with a median duration of response still not reached [33,34].

\section{Pembrolizumab}

The updated 5-y results of the treatment-naive cohorts from the seminal KEYNOTE-001 trial are the last evidence of long-term efficacy of pembrolizumab as monotherapy for the frontline treatment of NSCLC, regardless of PD-L1 
status (in a population still selected for PD-L1 $\geq 1 \%$ ) $[14,22,23]$. When pembrolizumab was administered in first line, the mOS was 22.3 months and the 5-y OS rate reached 23\% [14]. Looking at the data of pembrolizumab in PD-L1 $\geq 50 \%$ (KEYNOTE 024) NSCLC patients [35], after a mFU of more than 2 years, the mOS, 1-y and 2-y OS rates rached 30 months, 70 and 51\%, respectively (Table 2) [36]. These results were consistently longer than those reported with chemotherapy (mOS of 14 months; 1-y and 2-y OS rates of 55 and 35\% respectively) [36].

When frontline pembrolizumab was combined to carboplatin and pemetrexed in nonsquamous histology (KEYNOTE 021), 57\% of patients were progression-free at 1-y after, compared with $37 \%$ of the carboplatinpemetrexed group, at a mFU of 18.7 months [37]. Moreover, in the same analysis, the 1-y OS rate of the combination strategy was 77\% and remained still high at 18 months (70\%) and confirmed to be superior to chemotherapy alone (1-y OS of 69\% and 18-months OS of 56\%) [37]. Notably, after five additional months of FU, the mOS was yet NR in the pembrolizumab plus chemotherapy arm, while in the chemotherapy arm was 21.1 months [38]. The findings from this Phase II study have subsequently been confirmed by the updated results from the Phase III KEYNOTE-189 study, on pembrolizumab plus pemetrexed and a platinum salt presented at ASCO 2019 [44]. After a $\mathrm{mFU}$ of 23 months, the mOS was doubled in the pembrolizumab combination compared with the chemotherapy arm (22 vs 11 months; HR: 0.65; 95\% CI: 0.45-0.70) with a 2-y OS rate of 46 versus $30 \%$.

\section{Atezolizumab}

In the first-line cohort of BIRCH trial (atezolizumab in NSCLC with PD-L1 $\geq 5 \%$ of TC/IC), mOS was 24 months with 1-y, 2-y and 30-month OS rates of 66, 50 and 43\%, respectively [40]. Similar results were observed with regard to FIR study [28].

\section{Durvalumab, tremelimumab}

A total of 64 NSCLC patients received durvalumab as first-line treatment in its early Phase study and 2-y OS in this group was $46 \%$ [29].

The Phase III MYSTIC trial compared durvalumab versus durvalumab plus tremelimumab (anti-CTLA-4) versus platinum-based doublet chemotherapy in untreated metastatic NSCLC. Despite the primary end point of superiority of durvalumab monotherapy and combination over chemotherapy in selected patients (PD-L1 expression $\geq 25 \%$ in TC) was not met, a clinically meaningful improvement in OS was observed with durvalumab alone, compared with chemotherapy, while the combination of durvalumab plus tremelimumab did not add additional benefit to durvalumab alone (2-y OS of $38 \%$ with durvalumab vs $35 \%$ with durvalumab plus tremelimumab vs $23 \%$ with chemotherapy) [41].

\section{Predictive biomarkers of long-term survival}

Many potential prognostic and predictive biomarkers are currently under investigation in NSCLC patients treated with ICBs [45]. Among the several biological and clinical predictors of the benefit derivable from ICBs, PD-L1 expression is proven to be robustly correlated with long-term effectiveness thus far (Table 3). Several meta-analyses have been performed on PD-L1 prognostic/predictive role in NSCLC, showing that higher PD-L1 expression is associated with a higher likelihood of response to ICBs regardless of its threshold [46-49].

Besides PD-L1, the updated analyses on long-term survivors enrolled in the main ICB clinical trials may provide insights toward novel potential clinical and molecular biomarkers of long-term efficacy, which are discussed in the following section.

PD-L1

Nivolumab

In CheckMate 003 study, the survival benefit of nivolumab was seen across all PD-L1 levels, with similar 5-y OS rates for patients with $<1 \%$ versus $\geq 1 \%$ PD-L1 expression (20 vs $23 \%$ ), reaching the $43 \%$ in patients with PD-L1 $\geq 50 \%$ (out of 13 treated patients) [13]. In CheckMate 017 and CheckMate 057 [19], 2-y OS rates resulted again proportional to PD-L1 expression, ranging from 22 and 25\% (squamous and nonsquamous histologies, respectively) in PD-L1 negative cohorts to 35 and $48 \%$ in PD-L1 $\geq 50 \%$ groups. When the data of the four nivolumab trials CheckMate 003, 063, 017 and 057 were pooled, 4-y OS rates in PD-L1 <1\% and $\geq 1 \%$ groups were 11 and 19\%, respectively [43].

Moving to nivolumab-ipilimumab combinations, the updated analysis of CheckMate 012 clearly demonstrated that increasing PD-L1 expression was associated with higher clinical activity and survival outcomes of the combi- 
Table 3. PD-L1 expression subgroup results in trials of immune checkpoint blockers in pretreated/treatment-naive advanced non-small-cell lung cancer with a minimum follow-up of 2 years.

\begin{tabular}{|c|c|c|c|c|c|c|c|}
\hline Trial [Ref.] & ICB & PD-L1 ${ }^{\dagger}$ & N pts & $2 y$-PFS & $2 y-O S$ & $3 y-0 s$ & $5 y-0 S$ \\
\hline \multirow[t]{3}{*}{ CheckMate 003 [13] } & Nivolumab & $<1 \%$ & 30 & - & - & - & $20 \%$ \\
\hline & & $\geq 1 \%$ & 38 & - & - & - & $23 \%$ \\
\hline & & $\geq 50 \%$ & 13 & - & - & - & $43 \%$ \\
\hline \multirow[t]{3}{*}{ CheckMate 017 [19] } & Nivolumab & $<1 \%$ & 54 & - & $22 \%$ & - & - \\
\hline & & $\geq 1 \%$ & 63 & - & $20 \%$ & - & - \\
\hline & & $\geq 50 \%$ & 17 & - & $35 \%$ & - & - \\
\hline \multirow[t]{3}{*}{ CheckMate 057 [19] } & Nivolumab & $<1 \%$ & 108 & - & $25 \%$ & - & - \\
\hline & & $\geq 1 \%$ & 123 & - & $37 \%$ & - & - \\
\hline & & $\geq 50 \%$ & 66 & - & $48 \%$ & - & - \\
\hline \multirow{2}{*}{$\begin{array}{l}\text { Pooled analysis of CheckMate } 017,057,063 \text { and } \\
003 \text { [43] }\end{array}$} & Nivolumab & $<1 \%$ & 192 & - & - & - & - \\
\hline & & $\geq 1 \%$ & 224 & - & - & - & - \\
\hline \multirow[t]{3}{*}{ KEYNOTE-001 (pretreated cohort) $[14,22,23]$} & Pembrolizumab & $<1 \%$ & 80 & - & $23 \%$ & $9 \%$ & $4 \%$ \\
\hline & & $1-49 \%$ & 142 & - & $26 \%$ & $17 \%$ & $13 \%$ \\
\hline & & $\geq 50 \%$ & 100 & - & $39 \%$ & $30 \%$ & $25 \%$ \\
\hline \multirow[t]{2}{*}{ KEYNOTE-010 [24] } & Pembrolizumab & $\geq 1 \%$ & 690 & - & - & $23 \%$ & - \\
\hline & & $\geq 50 \%$ & 290 & - & - & $35 \%$ & - \\
\hline \multirow[t]{4}{*}{ POPLAR [26] } & Atezolizumab & TC3 or IC3 & 24 & - & $42 \%$ & $38 \%$ & - \\
\hline & & $\mathrm{TC} 2 / 3$ or $\mathrm{IC} 2 / 3$ & 50 & - & $36 \%$ & $21 \%$ & - \\
\hline & & $\mathrm{TC} 1 / 2 / 3$ or $\mathrm{IC} 1 / 2 / 3$ & 93 & - & $36 \%$ & $18 \%$ & - \\
\hline & & $\mathrm{TCO}$ and ICO & 51 & - & $25 \%$ & $21 \%$ & - \\
\hline \multirow[t]{4}{*}{ OAK (ITT1225 cohort) [27] } & Atezolizumab & TC3 or IC3 & 89 & - & $43 \%$ & - & - \\
\hline & & $\mathrm{TC} 2 / 3$ or $\mathrm{IC} 2 / 3$ & 168 & - & $36 \%$ & - & - \\
\hline & & $\mathrm{TC} 1 / 2 / 3$ or $\mathrm{IC} 1 / 2 / 3$ & 347 & - & $32 \%$ & - & - \\
\hline & & TCO and ICO & 260 & - & $27 \%$ & - & - \\
\hline \multirow[t]{2}{*}{ FIR (pretreated cohorts) [28] } & Atezolizumab & $\mathrm{TC} 3$ or IC3 & 38 (cohort 2) & $20 \% \mathbb{I}$ & - & $43 \% \mathbb{I}$ & - \\
\hline & & & 8 (cohort 3) & NEI & - & $19 \% \mathbb{I}$ & - \\
\hline \multirow[t]{4}{*}{ CheckMate 012 [32,34] } & Nivolumab + CT & $<1 \%$ & 23 & - & - & $22 \%$ & - \\
\hline & & $\geq 1 \%$ & 23 & - & - & $22 \%$ & - \\
\hline & Nivolumab + Ipilimumab & $\geq 1 \%$ & 47 & $38 \%$ & $58 \%$ & - & - \\
\hline & & $\geq 50 \%$ & 13 & $54 \%$ & $62 \%$ & - & - \\
\hline \multirow[t]{3}{*}{ KEYNOTE-001 (treatment-naive cohort) $[14,22,23]$} & Pembrolizumab & $\geq 1 \%$ & 79 & - & - & $22 \%$ & - \\
\hline & & $1-49 \%$ & 52 & - & $37 \%$ & $28 \%$ & $16 \%$ \\
\hline & & $\geq 50 \%$ & 27 & - & $67 \%$ & $48 \%$ & $30 \%$ \\
\hline \multirow[t]{3}{*}{ BIRCH (treatment-naive cohort) [40] } & Atezolizumab & $\mathrm{TC} 2 / 3$ or $\mathrm{IC} 2 / 3$ & 138 & $20 \%$ & $50 \%$ & $43 \% \mathbb{I}$ & - \\
\hline & & TC2 or IC2 & 73 & $13 \%$ & $49 \%$ & $39 \% \mathbb{I}$ & - \\
\hline & & TC3 or IC3 & 65 & $28 \%$ & $52 \%$ & $48 \% \mathbb{I}$ & - \\
\hline FIR (treatment-naive cohort) [28] & Atezolizumab & TC3 or IC3 & 7 & $14 \% \mathbb{I}$ & - & $29 \% \mathbb{I}$ & - \\
\hline \multicolumn{8}{|c|}{$\begin{array}{l}\text { †When not specified, PD-L1 expression refers to the percentage of tumor cells only. } \\
\text { ¥Analysis conducted on pooled pembrolizumab doses cohorts after a median follow-up of } 42.6 \text { months. } \\
\text { \$Data reported are per immune-related response criteria per investigator assessment. } \\
\mathbb{I} \text { I0-month OS rate. } \\
\text { IC: Immune cell; ICB: Immune checkpoint blocker; mFU: Median follow-up; mOS: Median overall survival; mPFS: Median progression-free survival; N: Number of patients treated with } \\
\text { ICl; NE: Not estimable; NR: Not reached; NSCLC: Non-small-cell lung cancer; ORR: Objective response rate; OS: Overall survival; PD-L1: Programmed cell death-ligand 1; TC: Tumor } \\
\text { cell. }\end{array}$} \\
\hline
\end{tabular}

nation approach [34]. The response rate of 43\% observed across PD-L1 expression levels increased to 57 and $92 \%$ in patients with PD-L1 $\geq 1 \%$ and $\geq 50 \%$, respectively. Of note, OS rate at 1 year was $100 \%$ in $\geq 50 \%$ PD-L1 patients, compared with $87 \%$ in $\geq 1 \%$ PD-L1 patients. The 2 -y OS rates were $62 \%$ in PD-L1 $\geq 50 \%$ patients compared with $58 \%$ in $\mathrm{PD}-\mathrm{L} 1 \geq 1 \%$ patients, respectively. 
On the contrary, in the CheckMate 012 cohort of patients who were treated with nivolumab plus platinum-based doublet chemotherapy, survival outcomes were similar across tumor PD-L1 expression levels (both 3-y OS rates of $22 \%)$. This data suggest that PD-L1 expression has no predictive value in patients treated with the combination of nivolumab and chemotherapy.

\section{Pembrolizumab}

In KEYNOTE 001 study, PD-L1 $\geq 50 \%$ was associated with higher OS compared with PD-L1 $1-49 \%$ and PD$\mathrm{L} 1<1 \%$, in both treatment-naive and pretreated patients [14]. Looking at the pretreated cohort, PD-L1 $\geq 50 \%$ was associated with an mOS of 15.4 months and a 5-y OS rate of 25\%. Albeit PD-L1 $<1 \%$ and $1-49 \%$ groups shared similar mOS estimations (8.5 months), the 5-year OS was remarkably higher in the $1-49 \%$ subgroup (13 vs 3\%). In the treatment-naive group, PD-L1 $\geq 50 \%$ patients experienced mOS of 35.4 months and 5-y OS rate of $30 \%$, higher compared with PD-L1 1-49\% patients (mOS of 19.5 months and 5-y OS rate of 15.7\%), while survival analyses on PD-L1 $<1 \%$ patients were not performed due to the small cohort $(\mathrm{n}=12)$.

At last, KEYNOTE-010 trial confirmed the higher survival outcomes of $\mathrm{PD}-\mathrm{L} 1 \geq 50 \%$ pretreated patients compared with the overall PD-L1 $\geq 1 \%$ population (3-y OS of 35 vs 23\%) [24]. KEYNOTE-189 was the only trial with updated results on pembrolizumab plus chemotherapy according to PD-L1 expression. Similarly to nivolumab-chemotherapy combinations, the survival benefit observed with the combination of pembrolizumab plus pemetrexed and platinum occurred regardless of PD-L1 expression [44].

\section{Atezolizumab}

In Phase II POPLAR and Phase III OAK trials, investigating atezolizumab activity in pretreated NSCLC patients, the survival benefit of atezolizumab was observed across all PD-L1 expression levels, including patients with TC0 and IC0 $[26,27]$.

Moreover, in the updated analyses of OAK trial, the mutually exclusive analyses showed that the survival benefit in the TC2 3 or IC2/3 population was driven by TC3 or IC3 patients [27]. The TC2 or IC2 subgroup itself, therefore, did not experience survival benefit with atezolizumab. The greatest benefit was indeed observed in the TC3 or IC3 subgroup, where mOS was 20.5 months with 2-y OS of 43\%, compared with 14.3 months with 2-y OS of $32 \%$ in the TC1/2/3 or IC1/2/3 subgroups. TC0 and IC0 subgroup had an mOS of 11.8 months with 2 -y OS of $27 \%$.

In the updated analysis of BIRCH trial, encouraging long-term OS rate at 30 months was observed regardless of PD-L1 status, with a range of 39-48\% for 30-month OS rate in three PD-L1 subgroups (TC 0 or IC0, TC2 or IC2 and TC3 or IC3) [40].

\section{Durvalumab, tremelimumab}

Putting the threshold of PD-L1 'strong' positivity at $\geq 25 \%$ and gathering both pretreated and first-line patients, 2 -y OS rates in Phase I/II durvalumab trial resulted 36\% (PD-L1 $\geq 25 \%$ ) and 22\% (PD-L1 <25\%) [29].

OS results of the MYSTIC trial across PD-L1 expression levels were reported at ASCO 2019 by Rizvi et al. [50], confirming the OS improvement for durvalumab alone compared with chemotherapy in patients with PD-L1 $>25 \%$. Moreover, no clear linear trend of OS improvement with increasing PD-L1 expression levels was observed with durvalumab plus tremelimumab versus chemotherapy.

\section{Tumor mutational burden}

Most recently, several clinical trials and retrospective analyses on NSCLC have highlighted tumor mutational burden (TMB) as a novel predictive and/or prognostic biomarker. TMB is the total number of nonsynonymous mutations per megabase (mut/Mb) in TC generally performed on tumor tissue or circulating tumor DNA through nextgeneration sequencing [51,52]. TMB has been associated, independently of the PD-L1 expression, with improved clinical benefit and survival outcomes of ICBs [51]. Albeit the role of TMB has been mostly envisaged in trials involving nivolumab (alone or in combination with ipilimumab) [9,53], its potential in predicting long-term outcomes has been recently presented for the association of durvalumab and tremelimumab [41].

In Phase III MYSTIC trial exploratory analyses on the predictive and prognostic role of tissue and blood TMB (tTMB and bTMB) were performed in the all population with survival updates at 2 years [41,50]. Long-term survival rates in patients receiving durvalumab plus tremelimumab increased with the threshold used for the definition of TMB-high cases, narrowing in parallel the proportion of patients definable 'TMB high'. Patients with tTMB 
$\geq 10 \mathrm{mut} / \mathrm{Mb}$ experienced longer 2-y OS with the combination of durvalumab plus tremelimumab compared with durvalumab alone and to chemotherapy arms ( 42 vs 35 vs $23 \%$ ). Conversely, patients with $\mathrm{tTMB}<10 \mathrm{mut} / \mathrm{Mb}$ had similar 2 -y OS across treatment groups, with slightly higher results obtained in the chemotherapy arm (19 vs 22 vs $25 \%)$ [41].

In the subgroup of patients with matched blood and tissue samples, bTMB showed good correlation with tTMB and subsequent analyses were conducted at different thresholds (cut-off of $\geq 16$ mut/Mb and $\geq 20 \mathrm{mut} / \mathrm{Mb}$ ), the OS estimations incrementally improving with increasing bTMB cut-offs for durvalumab plus tremelimumab. In the subgroup of patients with $\mathrm{bTMB} \geq 20$ mut/Mb, 2-y OS rates were 48,34 and $19 \%$ in durvalumab plus tremelimumab, durvalumab and chemotherapy arms, respectively. On the other hand, patients with bTMB $<20 \mathrm{mut} / \mathrm{Mb}$ had similar survival outcomes, regardless of treatment regimen (2-y OS of 20 to 27\%).

Of note, the first survival results with long-term FU based on the combined analysis of PD-L1 and bTMB were reported [50]. On one hand, durvalumab plus tremelimumab improved OS compared with chemotherapy across PD-L1 TC levels in patients with bTMB $\geq 16$ or 20 mut/Mb, but not in lower thresholds ( $2 \mathrm{y}$-OS ranging from 40 to $56 \%$ in $\mathrm{bTMB} \geq 20 \mathrm{mut} / \mathrm{Mb}$ vs $20-23 \%$ in $\mathrm{bTMB}<20 \mathrm{mut} / \mathrm{Mb}$ ).

\section{T-effector gene signature}

In the updated analysis of the OAK study, von Pawel et al. assessed the expression of the T-effector gene signature (CD274, IFNG and CXCL9) - which is a marker of pre-existing immunity - in long-term survivors, defined as having an $\mathrm{OS} \geq 2$ years [54]. These patients were shown to harbor higher $\mathrm{T}$ effector gene signature expression compared with non-long-term survivors enrolled in the study. In long-term survivors, median T-effector gene signature expression was 1.7-fold higher than non-long-term survivors in the atezolizumab arm (1.61 vs 2.40). This finding was consistent with previous data reported on atezolizumab monotherapy [55] and the more recent data on the combination of atezolizumab plus chemotherapy [8].

Taken together, these results suggest that markers of pre-existing immunity, similarly to TMB, could be a novel biomarker of clinical benefit of ICBs.

\section{Discussion}

Due to the positive results of a series of recent clinical trials incorporating ICBs administration in the first-line NSCLC treatment, several therapeutic options are going to be available in this setting in the future [56]. The FU of these studies is still too short to figure out the long-term effectiveness of novel strategies, mainly represented by combinatorial ones. Nevertheless, the availability of predictors of long-lasting efficacy of anti-PD-1/PD-L1 agents, when administered as monotherapies in both untreated and pretreated populations, may add relevant information to better address NSCLC patients to ICBs.

The survival plateau observed in the updates of ICB trials globally proved that approximately 15 and $25 \%$ of previously treated and untreated patients, respectively, are alive at 5 years from treatment beginning (Tables $1 \&$ 2). This proportion can meaningfully be enriched by selecting for PD-L1 expression (Table 3).

The recent potential recognition of cure in the metastatic setting of NSCLC patients is appealing, especially considering that the 5-y OS rates were stuck at 5\% in the pre-immunotherapy era. In this sense, for each treatment strategy the a priori identification of patients who are meant to be alive at long-term time-points is crucial for two main reasons: in order to reduce exposure to ineffective strategies (i.e., increase efficacy) and to avoid overtreatment (i.e., decrease toxicity). With the exception of the recent evidence about TMB predictive role in the case of PD1/PD-L1 and CTLA-4 concomitant inhibition, the most relevant biomarker is represented by PD-L1 expression. More recent evidence vouches moreover for the integration of diverse (and not correlated) biomarkers such as PD-L1 and TMB in addressing the long-lasting success of immunotherapy.

Due to the complex interface between the tumor and the immune system, the only PD-L1 evaluation could be diminishing, even if envisaging its potential in predicting long-term outcomes. More probably, the tumor and the host elements that concur to determine ICBs' activity participate in multifaceted interplays in determining ICBs' prolonged effects [57]. The dynamic nature of these forces may, in addition, be responsible of the differential duration of ICBs' benefit, still in the setting of long-lasting survival in NSCLC. Indeed, among the pretreated patients who drive prolonged OS benefit ( $\geq 2$ years [12]) further successful fate is obtained by a quote only. From the second to the third year since beginning treatment, a modest drop in survival rates can still be recognized, whereas from the third to the fifth year a true plateau, still with some death events, can be recognized (Table 1). 


\section{Conclusion}

The available updates of ICB studies are witness for their unprecedented long-term positive results in a critical disease setting such as advanced NSCLC. Five-year survival data are now robust enough to allow these prolonged positive outcomes to be envisaged, even more with the upcoming incorporation of immunotherapy in combinatorial, synergistic treatment strategies. In this sense, the appropriate patient selection and biomarker identification will be crucial, together with the integration of multimodal approaches to precise disease presentations, in order to potentially achieve cure in an increasing number of patients.

\section{Financial \& competing interests disclosure}

Marcello Tiseo declares: Advisory boards and speakers' fee for Astra-Zeneca, Pfizer, Eli-Lilly, BMS, Novartis, Roche, MSD, Boehringer Ingelheim, Otsuka, Takeda, Pierre Fabre; Research Grants from: Astra-Zeneca, Boehringer Ingelheim. The authors have no other relevant affiliations or financial involvement with any organization or entity with a financial interest in or financial conflict with the subject matter or materials discussed in the manuscript apart from those disclosed.

No writing assistance was utilized in the production of this manuscript.

\section{References}

1. Borghaei H, Paz-Ares L, Horn L et al. Nivolumab versus docetaxel in advanced nonsquamous non-small-cell lung cancer. N. Engl. J. Med. 373, 1627-139 (2015).

2. Brahmer J, Reckamp KL, Baas P et al. Nivolumab versus docetaxel in advanced squamous-cell non-small-cell lung cancer. N. Engl. J. Med. 373, 123-135 (2015).

3. Herbst RS, Baas P, Kim DW et al. Pembrolizumab versus docetaxel for previously treated, PD-L1- positive, advanced non-small-cell lung cancer (KEYNOTE-010): a randomized controlled trial. Lancet 387(10027), 1540-1550 (2016).

4. Rittmeyer A, Barlesi F, Waterkamp D et al. Atezolizumab versus docetaxel in patients with previously treated non-small-cell lung cancer (OAK): a phase 3, open-label, multicentre randomised controlled trial. Lancet 389(10066), 255-265 (2017).

5. Reck M, Rodríguez-Abreu D, Robinson AG et al. Pembrolizumab versus chemotherapy for pd-11-positive non-small-cell lung cancer. $N$. Engl. J. Med. 375(19), 1823-1833 (2016).

6. Gandhi L, Rodríguez-Abreu D, Gadgeel S et al. Pembrolizumab plus chemotherapy in metastatic non-small-cell lung cancer. N. Engl. J. Med. 378(22), 2078-2092 (2018).

7. Paz-Ares L, Luft A, Vicente D et al. Pembrolizumab plus chemotherapy for squamous non-small-cell lung cancer. N. Engl. J. Med. 379(21), 2040-2051 (2018).

8. Socinski MA, Jotte RM, Cappuzzo F et al. Atezolizumab for first-line treatment of metastatic nonsquamous nsclc. $N$. Engl. J. Med. 378(24), 2288-2301 (2018).

9. Hellmann MD, Ciuleanu TE, Pluzanski A et al. Nivolumab plus ipilimumab in lung cancer with a high tumor mutational burden. $N$. Engl. J. Med. 378(22), 2093-2104 (2018).

10. Emens LA, Butterfield LH, Hodi FS, Marincola FM, Kaufman HL. Cancer immunotherapy trials: leading a paradigm shift in drug development. J. Immunother. Cancer 4, 42 (2016).

11. Pabani A, Butts CA. Current landscape of immunotherapy for the treatment of metastatic non-small-cell lung cancer. Curr. Oncol. 25(Suppl. 1), S94-S102 (2018).

12. Van Damme V, Govaerts E, Nackaerts K, Dooms C, Wauters I, Vansteenkiste J. Clinical factors predictive of long-term survival in advanced non-small-cell lung cancer. Lung Cancer 79(1), 73-76 (2013).

13. Gettinger S, Horn L, Jackman D et al. Five-year follow-up of nivolumab in previously treated advanced non-small-cell lung cancer: results from the CA209-003 study. J. Clin. Oncol. 36(17), 1675-1684 (2018).

14. Garon EB, Hellmann MD, Rizvi NA et al. Five-year overall survival for patients with advanced non-small-cell lung cancer treated with pembrolizumab: results from the phase I KEYNOTE-001 study. J. Clin. Oncol.(2019) doi:10.1200/JCO.19.00934 (Epub ahead of print).

15. Topalian SL, Hodi FS, Brahmer JR et al. Safety, activity, and immune correlates of anti-PD-1 antibody in cancer. N. Engl. J. Med. 366(26), 2443-2454 (2012).

16. Gettinger SN, Horn L, Gandhi L et al. Overall survival and long-term safety of nivolumab (anti-programmed death 1 antibody, BMS-936558, ONO-4538) in patients with previously treated advanced non-small-cell lung cancer. J. Clin. Oncol. 33(18), 2004-2012 (2015).

17. Rizvi NA, Mazières J, Planchard D et al. Activity and safety of nivolumab, an anti-PD-1 immune checkpoint inhibitor, for patients with advanced, refractory squamous non-small-cell lung cancer (CheckMate 063): a phase 2, single-arm trial. Lancet Oncol. 16(3), 257-265 (2015). 
18. Lena H, Rizvi NA, Wolf J et al. 137O: Nivolumab in patients (pts) with advanced refractory squamous (SQ) non-small-cell lung cancer (NSCLC): 2-year follow-up from CheckMate 063 and exploratory cytokine profling analyses. J. Thorac. Oncol. 11(4), S115-S116 (2016).

19. Horn L, Spigel DR, Vokes EE et al. Nivolumab versus docetaxel in previously treated patients with advanced non-small-cell lung cancer: two-year outcomes from two randomized, open-label, phase III Trials (CheckMate 017 and CheckMate 057). J. Clin. Oncol. 35(35), 3924-3933 (2017).

20. Vokes EE, Ready N, Felip E et al. Nivolumab versus docetaxel in previously treated advanced non-small-cell lung cancer (CheckMate 017 and CheckMate 057): 3-year update and outcomes in patients with liver metastases. Ann. Oncol. 29(4), 959-965 (2018).

21. Garon EB, Rizvi NA, Hui R et al. Pembrolizumab for the treatment of non-small-cell lung cancer. N. Engl. J. Med. 372(21), 2018-2028 (2015).

22. Leighl NB, Hellmann MD, Hui R et al. Pembrolizumab in patients with advanced non-small-cell lung cancer (KEYNOTE-001): 3-year results from an open-label, phase 1 study. Lancet Respir. Med. 7(4), 347-357 (2019).

23. Felip E, Hellmann MD, Hui R et al. 4-year overall survival for patients with advanced NSCLC treated with pembrolizumab: results from KEYNOTE-001. J. Clin. Oncol. 36(Suppl. 15), 9030 (2018).

24. Herbst RS, Garon EB, Kim D-W et al. LBA4. Long-term follow-up in the KEYNOTE-010 study of pembrolizumab (pembro) for advanced NSCLC, including in patients (pts) who completed 2 years of pembro and pts who received a second course of pembro. Ann. Oncol. 29(Suppl. 10), x39-x43 (2018).

25. Fehrenbacher L, Spira A, Ballinger $\mathrm{M}$ et al. Atezolizumab versus docetaxel for patients with previously treated non-small-cell lung cancer (POPLAR): a multicentre, open-label, phase 2 randomised controlled trial. Lancet 387(10030), 1837-1846 (2016).

26. Mazières J, Park K, Lewanski C et al. 136PD_PR. 3-year survival and duration of response in randomized phase II study of atezolizumab (atezo) vs docetaxel (doc) in 2L+ NSCLC (POPLAR). J. Thorac. Oncol. 13(4), S79 (2018).

27. Fehrenbacher L, von Pawel J, Park K et al. Updated efficacy analysis including secondary population results for OAK: a randomized phase III study of atezolizumab versus docetaxel in patients with previously treated advanced non-small-cell lung cancer. J. Thorac. Oncol. 13(8), 1156-1170 (2018).

28. Spigel DR, Chaft JE, Gettinger S et al. FIR: Efficacy, safety, and biomarker analysis of a phase II open-label study of atezolizumab in PD-L1 - selected patients with NSCLC. J. Thorac. Oncol. 13(11), 1733-1742 (2018).

29. Antonia SJ, Balmanoukian A, Brahmer J et al. Clinical activity, tolerability, and long-term follow-up of durvalumab in patients with advanced NSCLC. J. Thorac. Oncoldoi:10.1016/j.jtho.2019.06.010 (2019) (Epub ahead of print).

30. Hellmann MD, Rizvi NA, Goldman JW et al. Nivolumab plus ipilimumab as first-line treatment for advanced non-small-cell lung cancer (CheckMate 012): results of an open-label, phase 1, multicohort study. Lancet Oncol. 18(1), 31-41 (2017).

31. Rizvi NA, Hellmann MD, Brahmer JR et al. Nivolumab in combination with platinum-based doublet chemotherapy for first-line treatment of advanced non-small-cell lung cancer. J. Clin. Oncol. 34(25), 2969-2979 (2016).

32. Juergens R, Hellmann M, Brahmer J et al. OA 17.03. First-line nivolumab plus platinum-based doublet chemotherapy for advanced NSCLC: CheckMate 012 3-year update. J. Thorac. Oncol. 12(11), S1792-S1793 (2017).

33. Antonia $S$, Gettinger $S$, Borghaei $\mathrm{H}$ et al. P1.01-02. Long-term outcomes with first-line nivolumab plus ipilimumab in advanced NSCLC: 3-year follow-up from CheckMate 012. J. Thorac. Oncol. 13(10), S458 (2018).

34. Goldman JW, Antonia SJ, Gettinger SN et al. Nivolumab (N) plus ipilimumab (I) as first-line (1L) treatment for advanced (adv) NSCLC: 2-yr OS and long-term outcomes from CheckMate 012. J. Clin. Oncol. 35(Suppl. 15), 9093 (2017).

35. Reck M, Rodríguez-Abreu D, Robinson AG et al. Pembrolizumab versus chemotherapy for PD-L1-positive non-small-cell lung cancer. N. Engl. J. Med. 375(19), 1823 (2016).

36. Reck M, Rodríguez-Abreu D, Robinson AG et al. Updated analysis of KEYNOTE-024: Pembrolizumab versus platinum-based chemotherapy for advanced non-small-cell lung cancer with PD-L1 tumor proportion score of 50\% or greater. J. Clin. Oncol. 37(7), 537-546 (2019).

37. Borghaei H, Langer CJ, Gadgeel S et al. LBA49. Updated results from KEYNOTE-021 cohort G: A randomized, phase 2 study of pemetrexed and carboplatin (PC) with or without pembrolizumab (pembro) as first-line therapy for advanced nonsquamous NSCLC. Ann. Oncol. 28(Suppl. 5), v605-v649 (2017).

38. Borghaei H, Langer CJ, Gadgeel S et al. 24-month overall survival from KEYNOTE-021 cohort G: Pemetrexed and carboplatin with or without pembrolizumab as first-line therapy for advanced nonsquamous non-small-cell lung cancer. J. Thorac. Oncol 14(1), 124-129 (2019).

39. Peters S, Gettinger S, Johnson ML et al. Phase II trial of atezolizumab as first-line or subsequent therapy for patients with programmed death-ligand 1-selected advanced non-small-cell lung cancer (BIRCH). J. Clin. Oncol. 35(24), 2781-2789 (2017).

40. Carcereny E, Felip E, Reck M et al. OA 17.02. Updated efficacy results from the BIRCH study: First-line atezolizumab therapy in PD-L1-selected patients with advanced NSCLC. J. Thorac. Oncol. 12(11), S1791-S1792 (2017). 
41. Peters $\mathrm{S}$, Cho BC, Reinmuth N et al. Tumor mutational burden as a biomarker of survival in metastatic non-small-cell lung cancer: Blood and tissue TMB analysis from MYSTIC, a phase III study of first-line durvalumab+tremelimumab vs chemotherapy. Cancer Res. 79(13 Suppl.), Abstract CT074 (2019).

42. Zhao X, Suryawanshi S, Hruska M et al. Assessment of nivolumab benefit-risk profile of a $240-\mathrm{mg}$ flat dose relative to a 3-mg/ $\mathrm{kg} \mathrm{dosing}$ regimen in patients with advanced tumors. Ann. Oncol. 28(8), 2002-2008 (2017).

43. Brahmer J, Borghaei H, Ramalingam SS et al. Long-term survival outcomes with nivolumab (NIVO) in pts with previously treated advanced non-small-cell lung cancer (NSCLC): Impact of early disease control and response. Cancer Res.79(13 Suppl.), Abstract CT195 (2019).

44. Gadgeel SM, Garassino MC, Esteban E et al. KEYNOTE-189: Updated OS and progression after the next line of therapy (PFS2) with pembrolizumab (pembro) plus chemo with pemetrexed and platinum vs placebo plus chemo for metastatic nonsquamous NSCLC. $J$. Clin. Oncol. 37(Suppl.), Abstract 9013 (2019).

45. Prelaj A, Tay R, Ferrara R et al. Predictive biomarkers of response for immune checkpoint inhibitors in non-small-cell lung cancer. Eur. J. Cancer 106, 144-159 (2019).

46. Wang A, Wang HY, Liu Y et al. The prognostic value of PD-L1 expression for non-small-cell lung cancer patients: a metaanalysis. Eur. J. Surg. Oncol. 41(4), 450-456 (2015).

47. Abdel-Rahman O. Correlation between PD-L1 expression and outcome of NSCLC patients treated with anti-PD-1/PD-L1 agents: a meta-analysis. Crit. Rev. Oncol. Hematol. 101, 75-85 (2016).

48. Aguiar PN Jr, Santoro IL, Tadokoro $\mathrm{H}$ et al. The role of PD-L1 expression as a predictive biomarker in advanced non-small-cell lung cancer: a network meta-analysis. Immunotherapy 8, 479-488 (2016).

49. Kim J, Cho J, Lee MH et al. Relative efficacy of checkpoint inhibitors for advanced NSCLC according to programmed death-ligand-1 expression: a systematic review and network meta-analysis. Sci. Rep. 8(1), 11738 (2018).

50. Rizvi NA, Cho BC, Reinmuth $\mathrm{N}$ et al. Blood tumor mutational burden (bTMB) and tumor PD-L1 as predictive biomarkers of survival in MYSTIC: first-line durvalumab (D) \pm tremelimumab (T) versus chemotherapy (CT) in metastatic (m) NSCLC. J. Clin. Oncol. 37(Suppl. 15), 9016 (2019).

51. Berland L, Heeke S, Humbert $\mathrm{O}$ et al. Current views on tumor mutational burden in patients with non-small-cell lung cancer treated by immune checkpoint inhibitors. J. Thorac. Dis. 11(Suppl. 1), S71-S80 (2019).

52. Kaderbhaï C, Tharin Z, Ghiringhelli F. The role of molecular profiling to predict the response to immune checkpoint inhibitors in lung cancer. Cancers 11(2), pii: E201 (2019).

53. Peters $\mathrm{S}$, Creelan B, Hellmann MD et al. Impact of tumor mutation burden on the efficacy of first-line nivolumab in stage IVor recurrent non-small-cell lung cancer: an exploratory analysis of CheckMate 026. Cancer Res. 77(13 Suppl.), Abstract CT082 (2017).

54. von Pawel J, Bordoni R, Satouchi M et al. Long-term survival in patients with advanced non-small-cell lung cancer treated with atezolizumab versus docetaxel: results from the randomised phase III OAK study. Eur. J. Cancer. 107, 124-132 (2019).

55. Kowanetz M, Zou W, McCleland $\mathrm{M}$ et al. Pre-existing immunity measured by Teff gene expression in tumor tissue is associated with atezolizumab efficacy in NSCLC. J. Thorac. Oncol. 12, S1817-S1818 (2017).

56. Proto C, Ferrara R, Signorelli D et al. Choosing wisely first line immunotherapy in non-small-cell lung cancer (NSCLC): what to add and what to leave out. Cancer Treat. Rev. 75, 39-51 (2019).

57. Chen DS, Mellman I. Elements of cancer immunity and the cancer-immune set point. Nature 541(7637), 321-330 (2017). 
